\title{
An atlas for clinical target volume definition, including elective nodal irradiation in definitive radiotherapy of biliary cancer
}

\author{
SILVIA BISELLO ${ }^{1}$, MATTEO RENZULLI $^{2}$, MILLY BUWENGE $^{1}$, LUCIA CALCULLI $^{2}$, GIUSEPPINA SALLUSTIO $^{3}$, \\ GABRIELLA MACCHIA ${ }^{4}$, FRANCESCO DEODATO ${ }^{4}$, GIANCARLO MATTIUCCI $^{5}$, SILVIA CAMMELLI $^{1}$, \\ ALESSANDRA ARCELLI $^{1}$, LUCIA GIACCHERINI ${ }^{1}$, FRANCESCO CELLINI ${ }^{5}$, GIOVANNI BRANDI ${ }^{6}$, \\ SARA GUERRI $^{2}$, SAVINO CILLA $^{7}$, RITA GOLFIERI ${ }^{2}$, LORENZO FUCCIO $^{8}$, \\ ALESSIO G. MORGANTI ${ }^{1 *}$ and ALESSANDRA GUIDO ${ }^{1 *}$
}

\footnotetext{
${ }^{1}$ Radiation Oncology Unit, Department of Experimental, Diagnostic and Specialty Medicine, S. Orsola-Malpighi Hospital;

${ }^{2}$ Radiology Unit, Department of Diagnostic and Preventive Medicine, S. Orsola-Malpighi Hospital, I-40138 Bologna;

${ }^{3}$ Radiology Unit; ${ }^{4}$ Radiation Oncology Unit, Catholic University of The Sacred Heart, I-86100 Campobasso;

${ }^{5}$ Radiation Oncology Department, Sacred Heart Catholic University, I-00168 Rome;

${ }^{6}$ Department of Experimental, Diagnostic and Specialty Medicine, 'L. e. A. Seragnoli' Institute of

Hematology and Medical Oncology, S. Orsola-Malpighi Hospital, I-40138 Bologna;

${ }^{7}$ Medical Physics Unit 'Giovanni Paolo II' Foundation, Catholic University of the Sacred Heart, I-86100 Campobasso;

${ }^{8}$ Department of Medical and Surgical Sciences, University of Bologna, I-40138 Bologna, Italy
}

Received June 7, 2018; Accepted October 31, 2018

\section{DOI: $10.3892 / \mathrm{ol} .2018 .9774$}

\begin{abstract}
Radiotherapy (RT) is a treatment option for advanced biliary tract cancer (BTC), often combined with sequential and/or concurrent chemotherapy. The use of modern RT techniques requires accurate clinical target volume (CTV) definition and delineation. However, guidelines for CTV delineation in BTC are lacking. Therefore, the aim of this study was to propose a computer tomography (CT) atlas for CTV definition of BTC. We previously proposed guidelines to define the nodal CTV (CTV-N) in BTC. In this study, based on a literature analysis, we defined the margins to be added to the gross tumor volume (GTV; subclinical and microscopic disease) to define the primary tumor CTV (CTV-T). An abdominal contrast enhanced planning CT scan was performed on three different patients with unresectable intrahepatic cholangiocarcinoma (CC), extrahepatic CC and gallbladder cancer. The GTV and anatomical reference structures were outlined on CT images. Then, based on our guidelines, the CTV-T and CTV-N were delineated and merged
\end{abstract}

Correspondence to: Dr Milly Buwenge, Radiation Oncology Unit, Department of Experimental, Diagnostic and Specialty Medicine, S. Orsola-Malpighi Hospital, Via Massarenti 9, I-40138 Bologna, Italy

E-mail: mbuwenge@gmail.com

*Contributed equally

Key words: radiotherapy, biliary neoplasms, elective nodal irradiation, clinical target volume to define the final CTV in the three patients. An atlas, showing the defined CTV, was generated from the reference CT images to illustrate the CTV for intra-hepatic CC, extra-hepatic CC and gallbladder cancer. This atlas can be used as an aid for CTV definition in patients with BTC treated with modern RT techniques.

\section{Introduction}

Biliary tract cancers (BTC) are rare malignancies, representing $<1 \%$ of all human cancers (1). Incidence is low in Western countries with $\sim 0.3-3.5 / 100.000$ cases/year, while in Asia and particularly in China, Thailand, and both North and South Korea, it is higher due to an increased frequency of liver flukes (2,3). BTC arise from biliary epithelium and almost $90 \%$ of cases are adenocarcinomas. BTC are classified as: Intrahepatic cholangiocarcinomas (CCs), extrahepatic CC, and gallbladder carcinoma (GC).

Complete resection (R0) is the only potentially curative treatment option. However, due to the advanced disease at diagnosis, more than half of the patients are not surgical candidates (4).

Radiotherapy (RT) generally combined with concurrent and/or sequential chemotherapy is considered as a treatment option for locally advanced BTC by international guidelines $(4,5)$. In fact, several studies on these tumors demonstrated the efficacy of chemoradiation, sometimes followed by a brachytherapy (BT) boost for symptoms palliation, local control (LC) and overall survival (OS) improvement (6-9).

However, although RT is considered a treatment option for locally advanced BTC, there is no consensus on clinical target volume (CTV) definition. Recently, Marinelli et al (10) published a review on the incidence of metastases in the 
Table I. CTV for intrahepatic cholangiocarcinoma.

JSHBPS

Delineation type classification
Recommended margins

\section{Tumor delineation}

Intrahepatic cholangiocarcinoma

Lymph node group, nodes delineation

Hepatoduodenal ligament lymph nodes

Common hepatic artery lymph nodes

Para-aortic lymph nodes

Posterior pancreaticoduodenal lymph nodes

Left gastric artery lymph nodes

Lesser gastric curvature lymph nodes

Right paracardial lymph nodes

Left paracardial lymph nodes

\author{
$-$
}

12

8

16

13

7

3

1

2

\section{$\mathrm{GTV}+10 \mathrm{~mm}$ radially}

$10 \mathrm{~mm}$ margin around the segment of portal vein from the confluence between the right and left hepatic ducts and the upper border of the pancreas

$10 \mathrm{~mm}$ margin around the common hepatic artery $10 \mathrm{~mm}$ margin around the abdominal aorta, from the diaphragmatic aortic hiatus to the upper border of the origin of the inferior mesenteric artery

$10 \mathrm{~mm}$ around the posterior pancreaticoduodenal artery $10 \mathrm{~mm}$ around the trunk of the left gastric artery The area around the lesser curvature of the stomach The narrowed anatomic space identified between gastric cardia and the liver, extending posteriorly to the aorta and inferiorly to the lesser curvature LNs

The anatomic space defined medially by the gastric fundus, anteromedially by the visceral peritoneum, posteriorly by the spleen, superiorly by the hemi diaphragm, and inferiorly by the great curvature LNs

Lymph node nomenclature is based on the 3rd English Edition of Classification of biliary tract cancers established by the JSHBPS. The anatomical structures of interest and the abdominal vessels of reference were identified for each lymph node region. JSHBPS, Japanese Society of Hepato-Biliary-Pancreatic Surgery; CTV, clinical target volume; GTV, gross tumor volume.

different regional nodal stations of patients with BTC. Based on that analysis, we proposed guidelines for the definition of the nodal CTV (CTV-N) in intrahepatic CC, extrahepatic CC, and GC (10).

The aim of the presest study was to provide supplementary literature data concerning the microscopic spread of the primary lesion and to suggest a proper CTV definition of the primary tumor (CTV-T). Furthermore, our objective was to define a general CTV by merging these evidence-based CTV-T and CTV-N. Finally, we aimed to present an atlas for locally advanced BTC delineation.

\section{Materials and methods}

Nodal clinical target volume $(C T V-N)$. The definition of the CTV-N was described in our previous analysis (10) and is only briefly summarized in the present study in Tables I-III.

All lymph nodes nomenclature was based on the 3rd English Edition of the Classification of BTC established by the Japanese Society of Hepato-Biliary-Pancreatic Surgery (11). We included in the hepatoduodenal lymph nodes (LN), also those around both portal vein and hepatic artery (groups 12a1, $12 \mathrm{a} 2,12 \mathrm{p} 1$ and $12 \mathrm{p} 2$ ), for anatomic contiguity of the structures in the hepatic hilum. Furthermore, we assumed the posterior pancreaticoduodenal LN (group 13) being similar to retropancreatic LN. Finally, we respected the definition of left and right paracardial LNs based on the gastric LNs contouring Atlas, from Wo et al (12).

In this CTV-N delineation, a $10 \mathrm{~mm}$ margin of soft tissue around vessels, ligament and ducts was suggested, based on several literature data (12-15), without overlap with radiosensitive structures (duodenum, liver, small bowel, stomach). Only for para-cardials nodes and lesser gastric curvature nodes, the suggested target was defined without any further expansion to preserve the surrounding OARs.

\section{Primary tumor CTV}

Literature review. To define the CTV-T we studied the microscopic extension of different sub sites of biliary cancers based on available literature data.

Intrahepatic $C C$. Intrahepatic $\mathrm{CC}$ usually appears as a homogenous mass with irregular but well-defined margins (mass-forming type). Rarely, they present as small lesions with diffuse bile duct thickening (periductal infiltrating type) or can grow intraductally, showing only a duct ectasia with variable visible mass (intraductal type) (16). Based on the study of Bi et al (17), the microscopic extension from the macroscopic disease ranged from 0.4 to $8.0 \mathrm{~mm}$. From the comparison between pathological evaluation of the surgical specimens and radiological images, they concluded that the imaging-based gross tumor volume (GTV) should be 
Table II. CTV for extrahepatic cholangiocarcinoma.

JSHBPS

Delineation type

Tumor delineation

Extrahepatic cholangiocarcinoma

Lymph node group, nodes delineation

Hepatoduodenal ligament lymph nodes

Left gastric artery lymph nodes

Common hepatic artery lymph nodes

Para-aortic lymph nodes

classification
Recommended margins
$\mathrm{GTV}+25 \mathrm{~mm}$ on the proximal direction of the bile duct $+20 \mathrm{~mm}$ on the distal direction $+15 \mathrm{~mm}$ radially in all directions

$10 \mathrm{~mm}$ margin around the segment of portal vein from the confluence between the right and left hepatic ducts and the upper border of the pancreas

$10 \mathrm{~mm}$ around the trunk of the left gastric artery

$10 \mathrm{~mm}$ margin around the common hepatic artery

$10 \mathrm{~mm}$ margin around the abdominal aorta, from the diaphragmatic aortic hiatus to the upper border of the origin of the inferior mesenteric artery

Posterior pancreaticoduodenal lymph nodes

Anterior pancreaticoduodenal lymph nodes

Peri-choledochal nodes

$12 \mathrm{~b} 2$
$10 \mathrm{~mm}$ around the posterior pancreaticoduodenal artery $10 \mathrm{~mm}$ margin around the anterior pancreaticoduo denal artery

$10 \mathrm{~mm}$ margin around the choledochal duct

Lymph node nomenclature is based on the 3rd English Edition of Classification of biliary tract cancers established by the JSHBPS. The anatomical structures of interest and the abdominal vessels of reference were identified for each lymph node region. JSHBPS, Japanese Society of Hepato-Biliary-Pancreatic Surgery; CTV, clinical target volume; GTV, gross tumor volume.

Table III. CTV for gallbladder carcinoma.

\section{JSHBPS}

Delineation type classification
Recommended margins

Tumor delineation

Gallbladder carcinoma

Lymph node group, nodes delineation

Hepatoduodenal ligament lymph nodes

Posterior pancreaticoduodenal lymph nodes

Anterior pancreaticoduodenal lymph nodes

Peri-choledochal nodes

Cystic duct lymph nodes
GTV + $25 \mathrm{~mm}$ radially in hepatic direction + gallbladder residual volume

$10 \mathrm{~mm}$ margin around the segment of portal vein from the confluence between the right and left hepatic ducts and the upper border of the pancreas $10 \mathrm{~mm}$ margin around the abdominal aorta, from the diaphragmatic aortic hiatus to the upper border of the origin of the inferior mesenteric artery

$10 \mathrm{~mm}$ around the posterior pancreaticoduodenal artery $10 \mathrm{~mm}$ margin around the anterior pancreaticoduodenal artery

$10 \mathrm{~mm}$ margin around the choledochal duct

$10 \mathrm{~mm}$ around the cystic duct

Lymph node nomenclature is based on the 3rd English Edition of Classification of biliary tract cancers established by the JSHBPS. The anatomical structures of interest and the abdominal vessels of reference were identified for each lymph node region. JSHBPS, Japanese Society of Hepato-Biliary-Pancreatic Surgery; CTV, clinical target volume; GTV, gross tumor volume.

expanded by $9.8 \mathrm{~mm}$ to include any microscopic disease with $100 \%$ accuracy.
Extrahepatic CCs. Extrahepatic CC show different behavior based on the histological type. They can be described as 

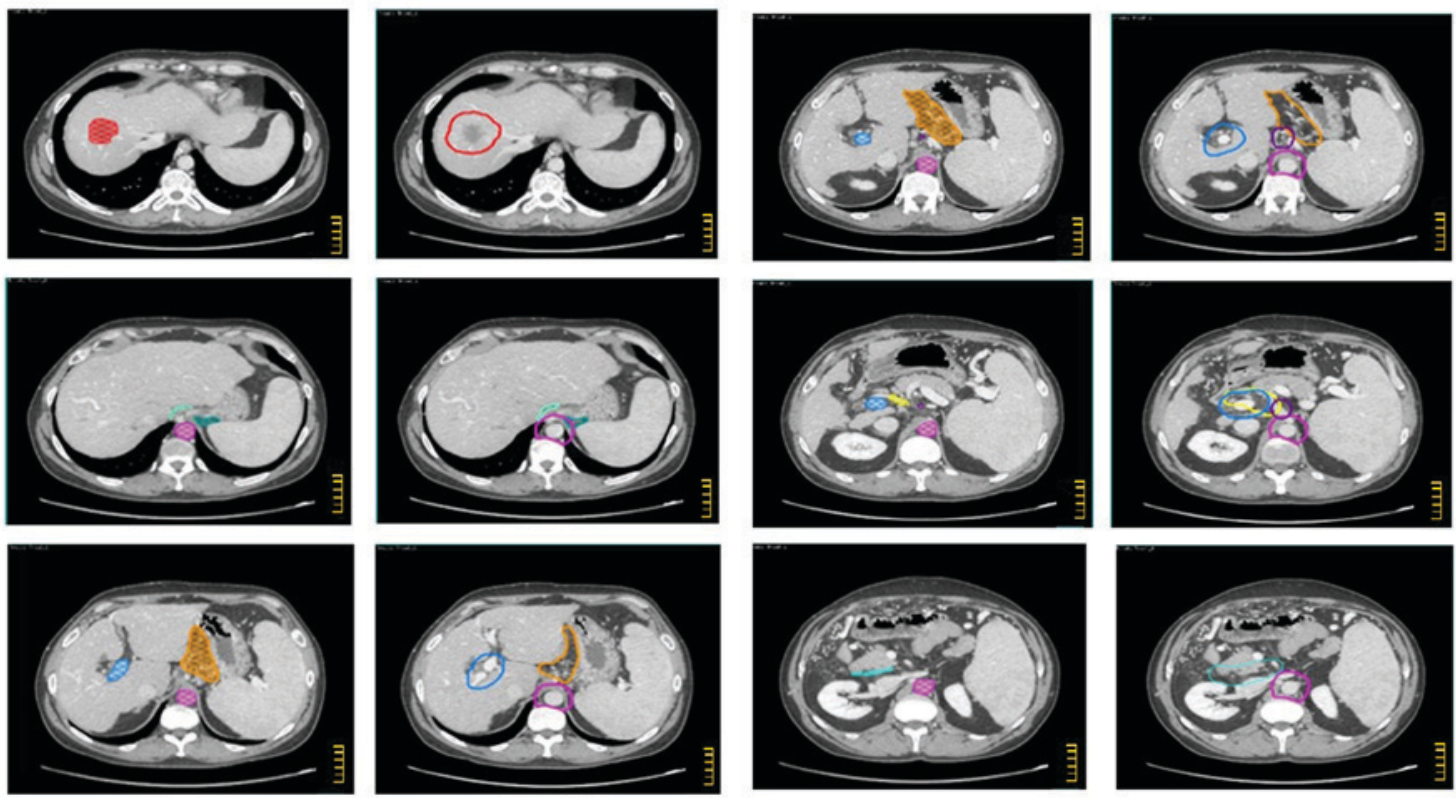

Figure 1. CTV definition for intrahepatic cholangiocarcinoma. Color wash structures: GTV (red), aorta (pink), left cardias (teal), right cardias (acquamarine), left gastric artery (purple), portal vein (light blue), common hepatic artery (yellow), lesser gastric curvature (orange), posterior pancreatico-duodenal artery (sky blue). Contoured structures: CTV (red), para-aortic LNs (purple), left para-cardial LNs (teal), right para-cardial LNs (acquamarine), left gastric artery LNs (purple), hepatoduodenal ligament (light blue), common hepatic artery LNs (yellow), lesser gastric curvature LNs (orange), posterior pancreatico-duodenal LNs (sky blue). The scale bar on the figures is at $1 \mathrm{~cm}$ interval.

papillary, nodular, and sclerosing type. Papillary types often show an intraluminal growth, whereas nodular and sclerosing types appear to spread longitudinally along the submucosal layer. Chang et al (18) reported a microscopic spread of papillary, nodular, and sclerosing types of 15.6, 10.0, and $15.6 \mathrm{~mm}$ in $90 \%$ of cases, respectively. For the latter two histological types, the length of microscopic tumor spread negatively correlates with macroscopic tumor size. Ebata et al (19) reported $10 \mathrm{~mm}$ intramural extension spread in all directions but observed that considering the superficial involvement, surgical margins of $20 \mathrm{~mm}$ could be assured to be negative proximally in $89.0 \%$ of cases and distally in $93.8 \%$.

Gallbladder carcinoma $(G C)$. GC presents an early invasion through the subserosal layer, due to the thin wall and lack of a muscolaris mucosae (20). Depending on the type of growth, Kondo et al (21) classified GC as: i) Hepatic bed type, where the mass penetrates through the gallbladder bed; ii) hepatic hilum type, with infiltration of the hepatic hilum from the neck of the gallbladder; iii) bed and hilum type, with massive invasion; iv) lymph node type, with only lymphatic involvement; v) cystic duct type and vi) localized type. There is lack of analyses in literature on microscopic extension evaluation. Only Ogura et al (20) demonstrated an average microscopic extension in most aggressive cases of advanced stage GC of $15.2 \mathrm{~mm}$ (range: 2 to $25 \mathrm{~mm}$ ).

Primary tumor CTV definition. Based on previously reported literature data, we defined the CTV-T for the three different sub sites.

Intrahepatic CC. Based on the study of Bi et al (17), for intrahepatic $\mathrm{CC}$ we added $10 \mathrm{~mm}$ radially to the GTV for CTV-T delineation (Table I).
Extrahepatic CC. Based on Chang et al (18) and Ebata et al data (19), our suggested CTV-T includes the GTV plus $25 \mathrm{~mm}$ proximally and $20 \mathrm{~mm}$ distally through the bile duct, and $15 \mathrm{~mm}$ radially in all other directions (Table II).

$G C$. Based on Ogura et al (20), for optimal control of microscopic disease we suggest including in the CTV-T the GTV plus $25 \mathrm{~mm}$ radially in the hepatic direction and any gallbladder residual volume (Table III).

Overall CTV definition. The definition of the overall CTV was achieved by merging the CTV-N as defined in our previous analysis (10), and the CTV-T as described in the previous paragraph. The CTV-N definition was performed by including for every sub site, all lymph node stations with an incidence of $5 \%$ of metastasis (10).

Atlas design. Three patients with locally advanced unresectable intrahepatic CC, extrahepatic CC, and GC were enrolled in this study. Three different CTVs were defined according to the method described above. Patients were immobilized in a customized Alpha Cradle. GTV was delineated using contrast enhanced planning computer tomography (CT) scans. Contouring of each anatomical structure close to the tumor was performed by experienced radiologists (MR, LC, GS, SG, RG) and radiation oncologists (GM, FD, GCM, FC, AG).

\section{Results}

The CTV-N, CTV-T and overall CTV are described in Tables I-III and shown in the atlas (Figs. 1-3) regarding intrahepatic $\mathrm{CC}$, extrahepatic $\mathrm{CC}$, and $\mathrm{GC}$, respectively. In the atlas, the GTV and the main anatomical structures 

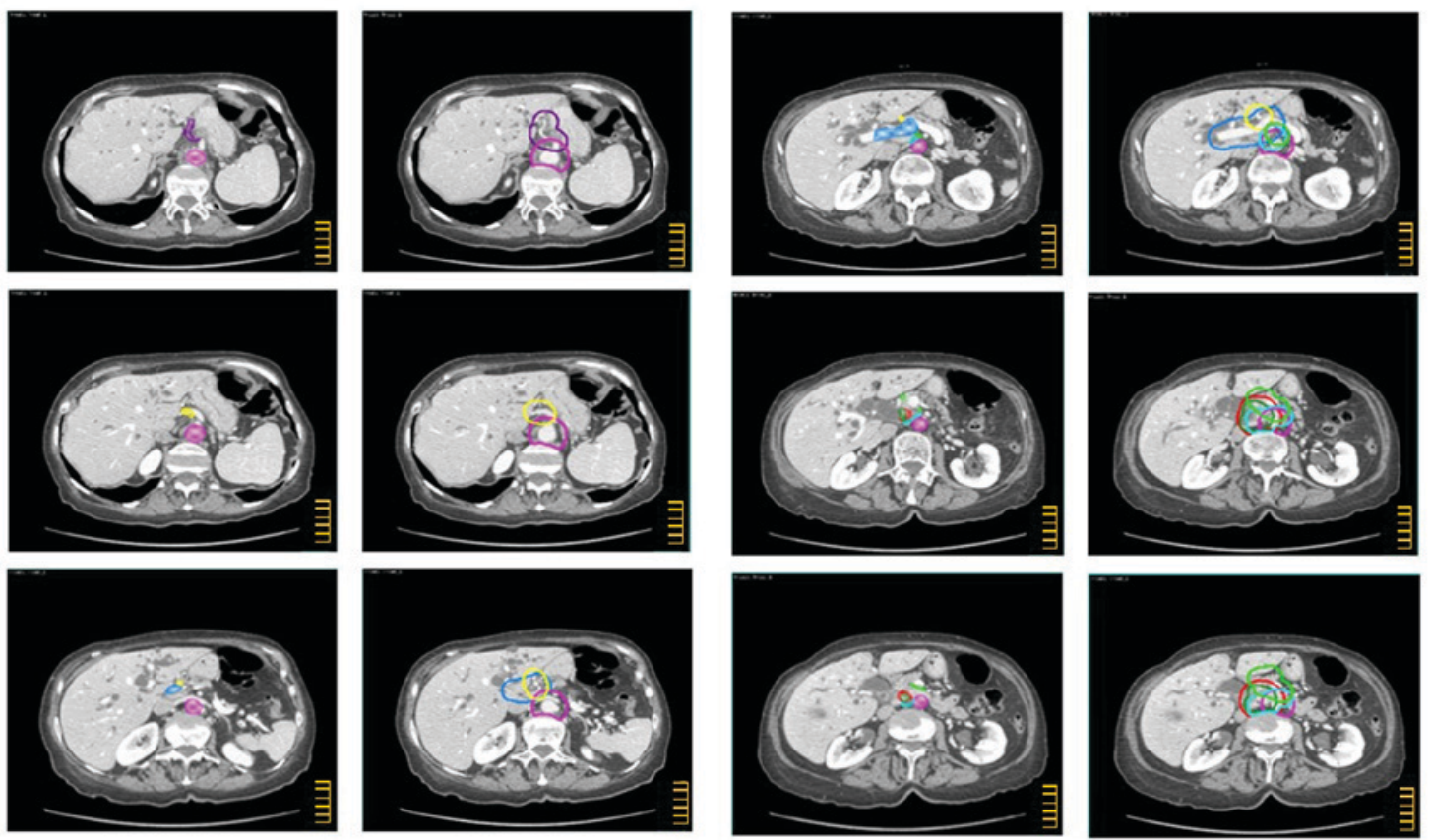

Figure 2. CTV definition for extrahepatic cholangiocarcinoma. Color wash structures: GTV (red), aorta (pink), left gastric artery (purple), portal vein (light blue), common hepatic artery (yellow), posterior pancreatico-duodenal artery (sky blue), anterior pancreatico-duodenal artery (green), choledochal duct (forest). Contoured structures: CTV (red), para-aortic LNs (purple), left gastric artery LNs (purple), hepatoduodenal ligament (light blue), common hepatic artery LNs (yellow), posterior pancreatico-duodenal LNs (sky blue), anterior pancreatico-duodenal LNs (green), pericholedochal LNs (forest). The scale bar on the figures is at $1 \mathrm{~cm}$ interval.
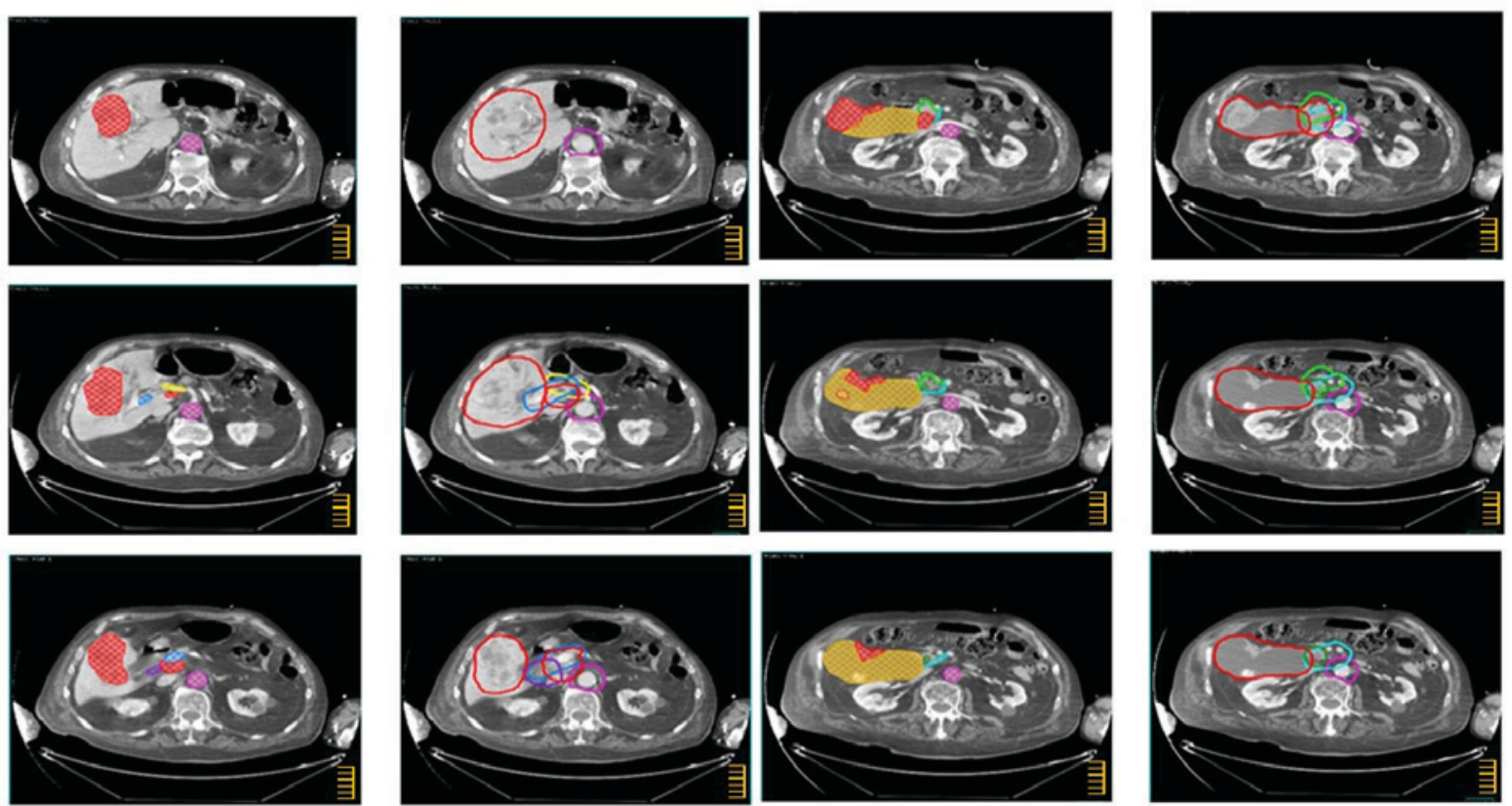

Figure 3. CTV definition for gallbladder carcinoma. Color wash structures: GTV (red), aorta (pink), portal vein (light blue), common hepatic artery (yellow), posterior pancreatico-duodenal artery (sky blue), anterior pancreatico-duodenal artery (green), choledochal duct (forest), cystic duct (slate blue), gallbladder (light orange), GTV_N (red). Contoured structures: CTV (red), para-aortic LNs (purple), hepatoduodenal ligament (light blue), common hepatic artery LNs (yellow), posterior pancreatico-duodenal LNs (sky blue), anterior pancreatico-duodenal LNs (green), pericholedochal LNs (forest), cystic duct LNs (slate blue), gallbladder (light orange), CTV_N (red). The scale bar on the figures is at $1 \mathrm{~cm}$ interval.

used as land marks are indicated with different colors. Furthermore, on the same figures, the different nodal sub sites and CTVs are also delineated with different colors. The legend of used colors and corresponding structures are shown in Fig. 4.

\section{Discussion}

Locally advanced unresectable disease is the most common presentation of BTC. Some studies demonstrated that combined-modality therapy based on chemoradiation +/-BT 


$\begin{array}{cl}1 & \text { Right Paracardial } \\ 2 & \text { Left Paracardial } \\ 3 & \text { Lesser Gastric Curvature } \\ 7 & \text { Left gastric artery } \\ 8 & \text { Common hepatic artery } \\ 12 & \text { Hepatoduodenal } \\ 12 \text { b2 } & \text { Pericholedochal } \\ 12 \mathrm{c} & \text { Cystic Duct } \\ 13 & \text { Posterior Pancreaticoduodenal } \\ 16 & \text { Para-aortic } \\ 17 & \text { Anterior Pancreaticoduodenal } \\ \text { GTV, CTV, GTV_N, CTV_N } \\ \text { Gallbladder }\end{array}$

Aquamarine
Teal
Orange
Purple
Yellow
Light Blue
Forest
Slate Blue
Sky Blue
Pink
Green
Red
Light Orange

Figure 4. Lymph node stations involved and color legend. Nodal stations numbers (indicated on the left side of the figure) are based on the 3rd English Edition of the Classification of BTC established by the Japanese Society of Hepato-Biliary-Pancreatic Surgery.

boost could reduce pain and improve LC, biliary decompression, and OS (6-9). In a recent retrospective study on 37 patients with unresectable extrahepatic CC treated with chemoradiation, 1-year LC and OS rates were 90 and 59\%, respectively (8).

Unfortunately, the results recorded in recent years (22-27) did not show a significant improvement of patients' outcome, which remain similar to those recorded in previous decades (6-9). An improvement in the clinical results could derive from innovative combinations with systemic therapies and/or from a more intensive use of most advanced RT techniques (IMRT, VMAT, IGRT). These could allow the delivery of higher RT doses without worsening radio-induced toxicity. However, these increasingly precise and conformed techniques require clearer guidelines for the contouring of the target and our atlas can represent a first proposal in this direction.

Our study presents some limitations mainly due to the paucity of data cancerning BTC microscopic and nodal spread. In fact, the information found in the analyzed studies were mainly generic, without differentiation based on tumor stage and sub-sites, nodal stage, and site of positive nodes. Therefore, we were unable to give specific indications about CTV definition based on the different stages and sub-sites of the three main BTCs. It represents a limitation of our results and an area for future work in this issue.

Obviously, the guidelines illustrated in this atlas should be adapted to individual patients. Particularly, careful attention must be given to inclusion in the target of any suspected or positive node, even if outside the proposed CTV.

Further pathological studies and sentinel node analysis would be useful to better understand the microscopic and lymphatic spread of these tumors based on tumor stage and nodal involvement.

The recommendations summarized in our atlas could be supplemented in future by pattern of failure studies where patients undergoing concurrent chemoradiation are closely followed using modern imaging techniques to define the most frequent sites of disease relapse. Studies of this type could lead to a more advanced version of the atlas.

We should underline that our study has been performed according to scientific literature where an atlas for target delineation is presented using the images of a single patient or as in this case, a few exemplary patients with different tumor sites. Clearly, this modality cannot give us information on the feasibility of large-scale application of the atlas. In particular, we cannot estimate the impact of this type of target contouring on OaRs irradiation. Therefore, further prospective studies testing feasibility and efficacy of this CTV delineation modality are justified.

In the last decade, there were no significant improvements in the outcome of patients with biliary tumors treated with chemoradiation. Therefore, innovative prospective trials are needed to improve clinical outcomes. This atlas, while providing practical guidelines for CTV delineation, could represent the basis to design these new studies.

\section{Acknowledgements}

The abstract has previously been presented as an oral communication at XXVII Congresso Nazionale Associazione Italiana Radioterapia Oncologia, Rimini, Italy, November 11-13, 2017 and at the 37th Annual meeting of the European Society for Radiotherapy \& Oncology, Barcelona, Spain, April 20-24, 2018 as an electronic poster.

\section{Funding}

No funding was received.

\section{Availability of data and materials}

All data generated or analyzed during this study are included in this published article. 


\section{Authors' contributions}

AGM and AG contributed to the conception and design of the study. SB, MB and AGM conducted the literature review. SB, AG, LF, MB and AGM wrote the manuscript. MR, LC, GS, SG and RG designed the atlas. AG, GCM, FD and GM defined the targets. SCa, AA, SCi and FD evaluated the images delineation. SCi, MB, AA, LG, LF, FC, GM and GB analyzed and interpreted the data. SCa, AA, LG, FC, GB and SCi critically revised the article for important intellectual content. All authors read and approved the final manuscript.

\section{Ethics approval and consent to participate}

All patients provided written informed consent for the use of their images. The study protocol was approved by the Ethics Committee of 'Giovanni Paolo II' Foundation, Catholic University of the Sacred Heart, Campobasso, Italy.

\section{Patient consent for publication}

Not applicable.

\section{Competing interests}

The authors declare that they have no competing interests.

\section{References}

1. Shaib T and El-Serag HB: The epidemiology of cholangiocarcinoma. Semin Liver Dis 24: 115-125, 2004.

2. Bragazzi MC, Cardinale V, Carpino G, Venere R, Semeraro R, GentileR, Gaudio Eand AlvaroD: Cholangiocarcinoma:Epidemiology and risk factors. Tranls Gastrointest Cancer 1: 21-32, 2012.

3. Siegel R, Ma J, Zou Z and Jemal A: Cancer statistics, 2014. CA Cancer J Clin 64: 9-29, 2014.

4. NCCN guideline version2018, Hepatobiliary Cancers.http://www nccn.org/professionals/physician_gls/default.aspx. Accessed March 17, 2018

5. Valle JW, Borbath I, Khan SA, Huguet F, Gruenberger T and Arnold D; ESMO Guidelines Committee: Biliary cancer: ESMO Clinical Practice Guidelines for diagnosis, treatment and follow-up. Ann Oncol 27 (Suppl 5): v28-v37, 2016.

6. Mattiucci GC, Autorino R, D'Agostino GR, Deodato F, Macchia G, Perri V, Tringali A, Morganti AG, Mutignani M and Valentini V: Chemoradiation and brachytherapy in extrahepatic bile duct carcinoma. Crit Rev Oncol Hematol 90: 58-67, 2014.

7. Deodato F, Clemente G, Mattiucci GC, Macchia G, Costamagna G, Giuliante F, Smaniotto D, Luzi S, Valentini V, Mutignani M, et al: Chemoradiation and brachytherapy in biliary tract carcinoma: Long-term results. Int J Radiat Oncol Biol Phys 64: 483-488, 2006.

8. Ghafoori AP, Nelson JW, Willett CG, Chino J, Tyler DS, Hurwitz HI, Uronis HE, Morse MA, Clough RW and Czito BG: Radiotherapy in the treatment of patients with unresectable extrahepatic cholangiocarcinoma. Int J Radiat Oncol Biol Phys 81: 654-659, 2011.

9. Chopra S, Mathew AS, Engineer R and Shrivastava SK: Positioning high-dose radiation in multidisciplinary management of unresectable cholangiocarcinomas: Review of current evidence. Indian J Gastroenterol 33: 401-407, 2014.

10. Marinelli I, Guido A, Fuccio L, Farioli A, Panni V, Giaccherini L, Arcelli A, Ercolani G, Brandi G, Cammelli S, et al: Clinical target volume in biliary carcinoma: A systematic review of pathological studies. Ant Res 37: 955-961, 2017.

11. Miyazaki M, Ohtsuka M, Miyakawa S, Nagino M, Yamamoto M, Kokudo N, Sano K, Endo I, Unno M, Chijiiwa K, et al: Classification of biliary tract cancers established by the Japanese Society of Hepato-Biliary-Pancreatic Surgery: 3(rd) English edition. J Hepatobiliary Pancreat Sci 22: 181-196, 2015.
12. Wo JY, Yoon SS, Guimaraes AR, Wolfgang J, Mamon HJ and Hong TS: Gastric lymph node contouring atlas: A tool to aid in clinical target volume definition in 3-dimensional treatment planning for gastric cancer. Pract Radiat Oncol 3: e11-e19, 2013.

13. Caravatta L, Sallustio G, Pacelli F, Padula GD, Deodato F, Macchia G, Massaccesi M, Picardi V, Cilla S, Marinelli A, et al: Clinical target volume delineation including elective nodal irradiation in preoperative and definitive radiotherapy of pancreatic cancer. Radiat Oncol 7: 86, 2012.

14. Taylor A, Rockall AG, Reznek RH and Powell ME: Mapping pelvic lymph nodes: Guidelines for delineation in intensity-modulated radiotherapy. Int J Radiat Oncol Biol Phys 63: 1604-1612, 2005.

15. Haijun Y, Qiuji W, Zhenming F, Yong H, Zhengkai L, Conghua X, Yunfeng $\mathrm{Z}$ and Yahua $\mathrm{Z}$ : A new approach to delineating lymph node target volumes for post-operative radiotherapy in gastric cancer: A phase II trial. Radiother Oncol 116: 245-251, 2015.

16. Chung YE, Kim MJ, Park YN, Choi JY, Pyo JY, Kim YC, Cho HJ, Kim KA and Choi SY: Varying appearances of cholangiocarcinoma: Radiologic-pathologic correlation. Radiographics 29: 683-700, 2009.

17. Bi AH, Zeng ZC, Ji Y, Zeng HY, Xu C, Tang ZY, Fan J, Zhou J, Zeng MS and Tan YS: Impact factors for microinvasion in intrahepatic cholangiocarcinoma: A possible system for defining clinical target volume. Int J Radiat Oncol Biol Phys 78: 1427-1436, 2010.

18. Chang YR, Lee KB, Jang JY, Lim CS, Kang MJ, Kwon W, Jung WH and Kim SW: Analysis of microscopic tumor spread patterns according to gross morphologies and suggestions for optimal resection margins in bile duct cancer. J Gastrointest Surg 18: 1146-1154, 2014.

19. Ebata T, Watanabe H, Ajioka Y, Oda K and Nimura Y: Pathological appraisal of lines of resection for bile duct carcinoma. Br J Surg 89: 1260-1267, 2002

20. Ogura Y, Tabata M, Kawarada Y and Mizumoto R: Effect of hepatic invasion on the choice of hepatic resection for advanced carcinoma of the gallbladder: Histologic analysis of 32 surgical cases. World J Surg 22: 262-267, 1998.

21. Kondo S, Nimura Y, Kamiya J, Nagino M, Kanai M, Uesaka K and Hayakawa N: Mode of tumor spread and surgical strategy in gallbladder carcinoma. Langenbecks Arch Surg 387: 222-228, 2002.

22. Baisden JM, Kahaleh M, Weiss GR, Sanfey H, Moskaluk CA, Yeaton P, de Lange EE and Rich TA: Multimodality treatment with helical tomotherapy intensity modulated radiotherapy, capecitabine, and photodynamic therapy is feasible and well tolerated in patients with hilar cholangiocarcinoma. Gastrintest cancer Res 2: 219-224, 2008.

23. Phelip JM, Vendrely V, Rostain F, Subtil F, Jouve JL, Gasmi M, Michel P, Le Malicot K, Smith D, Seitz JF, et al: Gemcitabine plus cisplatin versus chemoradiotherapy in locally advanced biliary tract cancer: Fédération francophone de cancérologie digestive 9902 phase II randomized study. Eur J Cancer 50: 2975-2982, 2014.

24. Autorino R, Mattiucci GC, Ardito F, Balducci M, Deodato F, Macchia G, Mantini G, Perri V, Tringali A, Gambacorta MA, et al: Radiochemotherapy with gemcitabine in unresectable extrahepatic cholangiocarcinoma: Long-term result of a phase II study. Anticancer Res 36: 737-740, 2016.

25. Lee KJ, Yi SW, Cha J, Seong J, Bang S, Song SY, Kim HM and Park SW: A pilot study of concurrent chemoradiotherapy with gemcitabine and cisplatin in patients with locally advanced biliary tract cancer. Cancer Chemother Pharmacol 78: 841-846, 2016.

26. Yi SW, Kang DR, Kim KS, Park MS, Seong J, Park JY, Bang SM, Song SY, Chung JB and Park SW: Efficacy of concurrent chemoradiotherapy with 5-fluorouracil or gemcitabine in locally advanced biliary tract cancer. Cancer Chemother Pharmacol 73: 191-198, 2014.

27. Chen SC, Chen MH, Li CP, Chen MH, Chang PM, Liu CY, Tzeng CH, Liu YM, Yen SH, Chao Y and Huang PI: External bean radiation therapy with or without concurrent chemotherapy for patients with unresectable locally advanced hilar cholangiocarcinoma. Hepatogastroenterology 62: 102-107, 2015.

This work is licensed under a Creative Commons Attribution-NonCommercial-NoDerivatives 4.0 International (CC BY-NC-ND 4.0) License. 